\title{
Investigation of Anaemia in Geriatric patients Admitted to Norfolk \& Norwich University teaching Hospital Old People's Medicine/Geriatric Department
}

Lamabadusuriya ${ }^{1}$, Emmanuel Tigalya ${ }^{2 *}$, and Yasmine Karachiwala ${ }^{3}$.

${ }^{1}$ Dilusha Consultant, Norflok \& Norwich University Teaching Hospital. UK.

${ }^{2}$ Locum SpR, Norflok \& Norwich University Teaching Hospital. UK.

${ }^{3}$ Clinical Fellow, Norflok \& Norwich University Teaching Hospital. UK.

*Corresponding author: Emmanuel Tigalya, Norflok \& Norwich University Teaching Hospital. UK.

Received date: October 05, 2021; Accepted date: October 12, 2021; Published date: October 20, 2021.

Citation: Lamabadusuriya, Emmanuel Tigalya, and Yasmine Karachiwala (2021), Investigation of Anaemia in Geriatric patients Admitted to Norfolk \& Norwich University teaching Hospital Old People's Medicine/Geriatric Department, J. New Medical Innovations and Research, 2(5): DOI: $10.31579 / 2767-7370 / 024$

Copyright: (C) 2021 Emmanuel Tigalya. This is an open-access article distributed under the terms of The Creative Commons Attribution License, which permits unrestricted use, distribution, and reproduction in any medium, provided the original author and source are credited.
Abstract: level tests. investigate to start treat if indicated.
i) Iron level check
ii) Vitamin B12 and folate check
iii) Ferritin

Aims: To assess if anaemia detected in geriatric patients admitted to the Old Peoples Medicine Department at Norfolk and Norwich University Teaching Hospital was investigated with appropriate laboratory tests- haematinics and iron

Also increase awareness among doctors of the high prevalence of anaemia in geriatric patients and the need to

Target population or scope: All patients aged 80 and above admitted under the care of NNUH OPM department on a given day incidentally found to have anaemia.

Criteria: All over 80 year olds admitted to hospital found incidentally to have low HB (anaemia) should be investigated by having elemental iron level and haematinic checks;

Target population or scope: All patients aged 80 and above admitted under the care of NNUH OPM department on a given day incidentally found to have anaemia.

Keywords: Anaemia; Geriatric patients; - haematinics; iron level tests

\section{Introduction}

\section{Aims}

To assess if anaemia detected in geriatric patients admitted to the Old Peoples Medicine Department at Norfolk and Norwich University Teaching Hospital was investigated with appropriate laboratory testshaematinics and iron level tests.
Also increase awareness among doctors of the high prevalence of anaemia in geriatric patients and the need to investigate to start treat if indicated.

\section{Criteria}

All over 80 year olds admitted to hospital found incidentally to have low $\mathrm{HB}$ (anaemia) should be investigated by having elemental iron level and haematinic checks;

iv) Iron level check 
v) Vitamin B12 and folate check

vi) Ferritin

Although the mean corpuscular volume (MCV) and red cell width (RDW) should guide which investigations should be ordered in anaemia +/macrocytosis, the accepted practice at NNUH is for haematinics to be ordered for all elderly anaemic patients on admission if not recently checked.

\section{Standards}

A standard is the proportion of patients who meet the criterion.

$98 \%$ of all anaemic geriatric patients should have haematinics and iron levels checks when the low $\mathrm{Hb}$ is detected. This can be arranged by GPs or during hospital admission.

A $2 \%$ slippage is allowed to account for patient who are anaemic but don't attend hospital regularly because they are well.

\section{Method}

From the OPM PAS list of 247, patient admission FBC- blood tests archived in the ICE database of NNUH hospital laboratory department belonging to anaemic over 80 year old patients were identified and

\begin{tabular}{|l|l|l|}
\hline Total Number of Anaemic Patients & $\begin{array}{l}\text { Number of Anaemic Patients } \\
\text { investigated further for anaemia }\end{array}$ & $\begin{array}{l}\text { Number of Anaemic Patients not } \\
\text { investigated anaemia further }\end{array}$ \\
\hline $\mathrm{N}$ & $94(56.6 \%)$ & $72(43.4 \%)$ \\
\hline
\end{tabular}

Table. 1. Proportions of Anaemic Patients in the Audit who were further investigated or not further investigated. Total of Anaemic patients (N) was 166.

\begin{tabular}{|l|l|l|}
\hline Criterion & $\begin{array}{l}\text { Number of Patient meeting } \\
\text { criteria }\end{array}$ & Percentage \\
\hline 1- had Iron level checked & 32 & $34.0 \%$ \\
\hline $\begin{array}{l}\text { 2- had Vitamin B12 and } \\
\text { folate checked }\end{array}$ & 45 & $47.9 \%$ \\
\hline 3- had Ferritin checked & 17 & $18.1 \%$ \\
\hline
\end{tabular}

Table .2. Number of Patients meeting each criterion

\section{Discussion}

Norwich \& Norfolk University Teaching Hospital has one of the largest Old People's Medicine department in UK with a capacity of over 300 inpatients at any given day and so this presented us with an excellent opportunity to audit the detection and management of anaemia in geriatric patients admitted to the hospital.

The elderly is an important demographic population that is growing globally especially in the western world, in this demographic of anaemia is now recognized as a risk factor for a number of adverse outcomes even when the anaemia is mild. Adverse outcomes include decreased physical performance, increased number of falls, increased frailty, decreased cognition, increased dementia, increased hospitalization and increased mortality [1-4].

The prevalence of anaemia is now known to increase with increasing age, reviewed to check if haematinics and elemental level tests were ordered by doctors after initial FBC tests indicated a low $\mathrm{Hb}$ (anaemia).

*OPM PAS list is the list of all admitted patients under the OPM department on a given day.

*Haematinics in this audit included only Vitamin B12, folate and Ferritin

Anaemia was defined based World Health Organization (WHO) - defined anaemia as (haemoglobin $<12 \mathrm{~g} / \mathrm{dL}$ in women and $<13 \mathrm{~g} / \mathrm{dL}$ in men).

\section{Results}

This is usually stated as the proportion meeting the criteria and compared that against the standard set.

Overall from our evaluation of the laboratory results of the 247 patients on the PAS list, 166 were found to be anaemic as opposed 81 who were not anaemic. Therefore $67 \%$ of patients admitted under OPM on a given day could be classed as anaemic.

Of the 166 anaemic patients only 94 patients (56\%) were further investigated under any of the 3 criteria, this is much below the standard set $(98 \%)$. Further analysis of the 94 investigated anaemic patients revealed that 32 (34\%) patients met criterion 1 (had iron level checks), 45 $(47.9 \%)$ patients met criterion 2 (had Vitamin B12\& Folate level checks) and $17(18.1 \%)$ patients met criterion 3 ( had Ferritin level checks). 


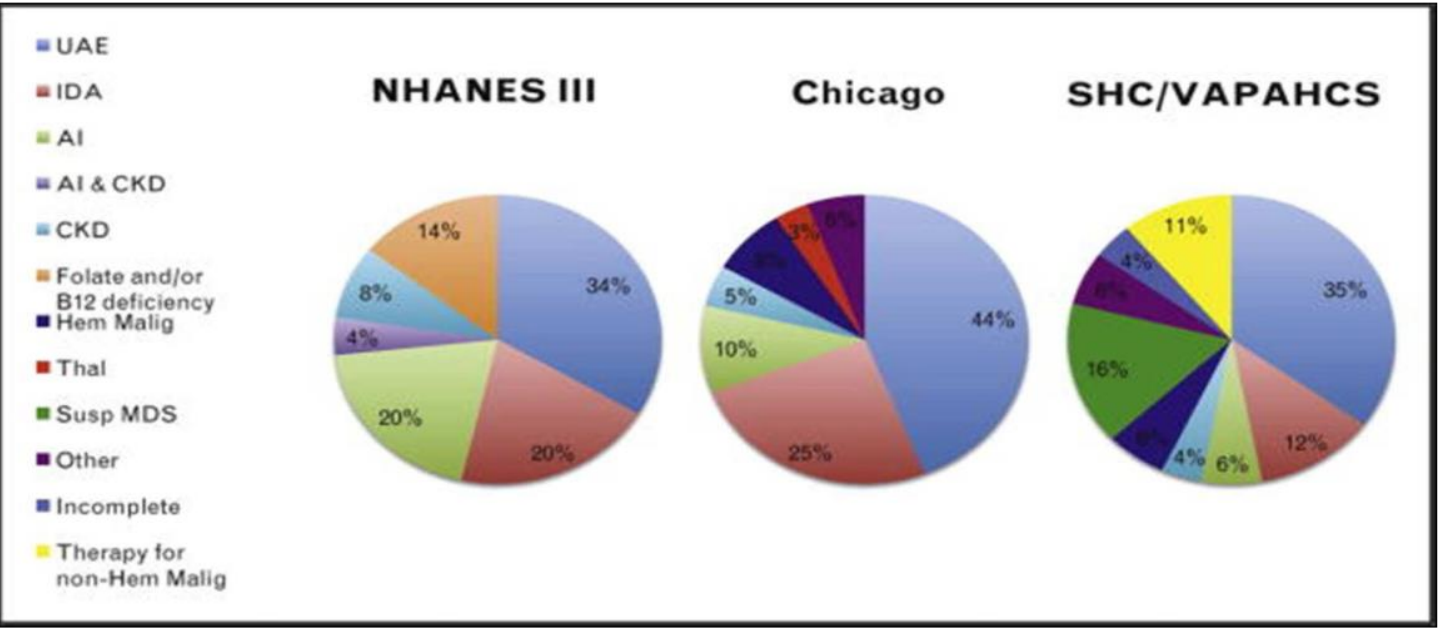

Figure 1. Prevalence of Anemia in the Elderly

Prevalence of anemia in the elderly by cause identified in three studies. Studies shown are National Health and Nutrition Survey (NHANES) III,8 Chicago,27 and Stanford Hospital \& Clinics, VA Palo Alto Health Care System (SHC/VAPAHCS).26 AI, anemia of inflammation; CKD, anemia secondary to renal disease; Hem Malig, hematologic malignancy; IDA, iron-deficiency anemia; Susp MDS, suspicious for myelodysplastic syndrome; Thal, thalassemia; UAE, unexplained anemia of the elderly.

Lack of iron is by far the most frequent nutritional deficiency anaemia. Similar to folate deficiency, iron depletion is often associated with malnutrition. Age dependent alterations in function of GI tract, polypharmacy, and social isolation may lead to malnutrition and subsequent anaemia. Up to $36 \%$ of a general geriatric medical population have absolute iron deficiency as a form nutritional deficiency.

In clinical practice it is important to consider bleeding due to a variety of medications (eg, acetylsalicylic acid, standard or direct oral anticoagulants) or GI diseases, including GI malignancy as a frequent cause of iron deficiency anaemia (IDA) in older patients through chronic blood loss.

Thus, apart from iron re- placement therapy, a careful GI diagnostic workup is mandatory to define a possible site of blood loss in these patients.

Iron-restricted erythropoiesis can cause Iron deficiency anaemia due to an absolute iron deficiency; i.e underlying problems in iron sequestration which is mediated by hepcidin; and/or a functional iron deficiency due to blunted erythropoietin-stimulated erythropoiesis.

Deficiency in Vitamin B12 or folate can also result in deficiency anaemias. The cause may be due to dietary deficiency in malnutrition, malabsorption, or lack of folate supplementation for example in pregnancy. Malnutrition in association with alcohol abuse may result in folate deficiency. Medication such like anticonvulsants and methotrexate can also cause folate deficiency.

Pernicious anaemia is a classic example of vitamin B12 deficiency anaemia which can be accompanied by neurological symptoms that resolve with vitamin B 12 replacement. Helicobacter pylori infection, acid-reducing drugs such as omeprazole and atrophic gastritis may cause hypochlorhydria leading to a food-cobalamin malabsorption syndrome, for this reason including folate and B-12 measurement in basic laboratory screening for elderly anaemic patients is important.

In anaemic patients with chronic inflammation, when serum ferritin and transferrin saturation values are inconclusive and there is a lack of response to iron therapy, a diagnosis of the anaemia of inflammation or unexplained anaemia of the elderly (UAE) can be given. Aging has been associated with increasing levels of IL 6 which in turn correlates with anaemia, this is particularly seen elderly patients with multiple comorbidities.

Clinical evaluation for inflammatory conditions, measurement of Creactive protein, fibrinogen, erythrocyte sedimentation rate (ESR), IL6, and hepcidin levels helps in establishing the diagnosis. Management of chronic inflammatory anaemia requires addressing underlying conditions and supplementation with an erythropoiesis stimulating agent (ESA) sometimes [1-4].

Anaemia related to chronic kidney disease (CKD) is due to reduced erythropoeitin production. Serum creatinine and GFR must be determined in order to evaluate for $\mathrm{CKD}$, in which case referral to a nephrologist may be appropriate. The suggested cut-off of glomerular filtration rate (GFR) is $<60 \mathrm{~mL} / \mathrm{min}$ for consideration that the anaemia is secondary renal disease.

With unexplained anaemia of the elderly (UAE) also referred to as idiopathic anaemia, diagnosis is usually considered when other causes of anaemia in the elderly have been eliminated. The diagnosis of UAE is based on the findings of a hypoproliferative anaemia: a low reticulocyte index and an inadequate erythropoietin level for the degree of anaemia. The management of these patients is a serious and recurrent issue, since the aetiology is usually not understood, there is no proven efficacious intervention except transfusion therapy when symptomatic [1-4].

It is estimated that UAE occurs in approximately $20-30 \%$ of communitydwelling elderly anaemic subjects in cross sectional epidemiologic studies and in up to half of anaemic nursing home residents.

Mechanisms suspected to underly UAE include hypogonadism (79), alterations in haematopoietic stem and erythroid progenitor cell number and/or function, and the presence of "early" or overt myelodysplastic syndrome (MDS).

A percentage of those with otherwise unexplained anaemia are likely to have MDS.

MDS is a disease of the elderly, with the median age of diagnosis in the late 60 s to $70 \mathrm{~s}$.

In one study 37 of $245(15 \%)$ hospitalised geriatric patients who underwent evaluation based on the French-American-British (FAB) classification for unexplained haematologic abnormalities were diagnosed with MDS, also using the FAB classification [1-4]. 
The criteria selected in this audit were guided by NICE guidelines on anaemia [5].

NICE recommends that if the diagnosis of iron deficiency anaemia is in doubt despite serum ferritin test results, diagnostic trials of iron treatment in can be considered.

NICE guidelines also recommend Vitamin B12 and folate level checks, and this should be done especially when a person has normocytic anaemia with a low or normal ferritin level.

Serum ferritin is perhaps the most frequently used peripheral blood test for assessing iron deficiency anaemia. Iron stores are reliably depleted when ferritin is less than $12 \mathrm{mg} / \mathrm{L}$. However, whereas a low serum ferritin dependably indicates iron deficiency, a normal level in an elderly patient does not necessarily rule out iron deficiency, as serum ferritin rises with age.

Iron indices may also be influenced by the presence of inflammation, in which the transferrin is normal or low, and the ferritin is normal or elevated.
In one study of the elderly, the sTfR(soluble transferrin receptor)/log ferritin was found to be much more sensitive than standard iron indices in diagnosing iron deficiency anaemia. While the serum ferritin is the most cost-effective diagnostic measurement of iron deficiency, the sTfR/log ferritin may be useful however, the lack of standardised reagents for the sTfR complicates local interpretation of the sTfR/ferritin ratio for many diagnostic laboratories.

It has been suggested that a carefully monitored trial of iron supplementation can be used to confirm the diagnosis IDA if the anaemia resolves following a period of supplementation.

Given the above difficulties, elemental iron level checks are effective and reliable in diagnosing IDA in my opinion.

Good workup of anaemia must also consider unexpected diagnoses including chronic kidney disease (CKD) or occult malignancy. If absolute iron deficiency is diagnosed, in the elderly and post-menopausal population it is mandatory to rule out gastrointestinal pathology, including malignancy (Appendix A, 1).

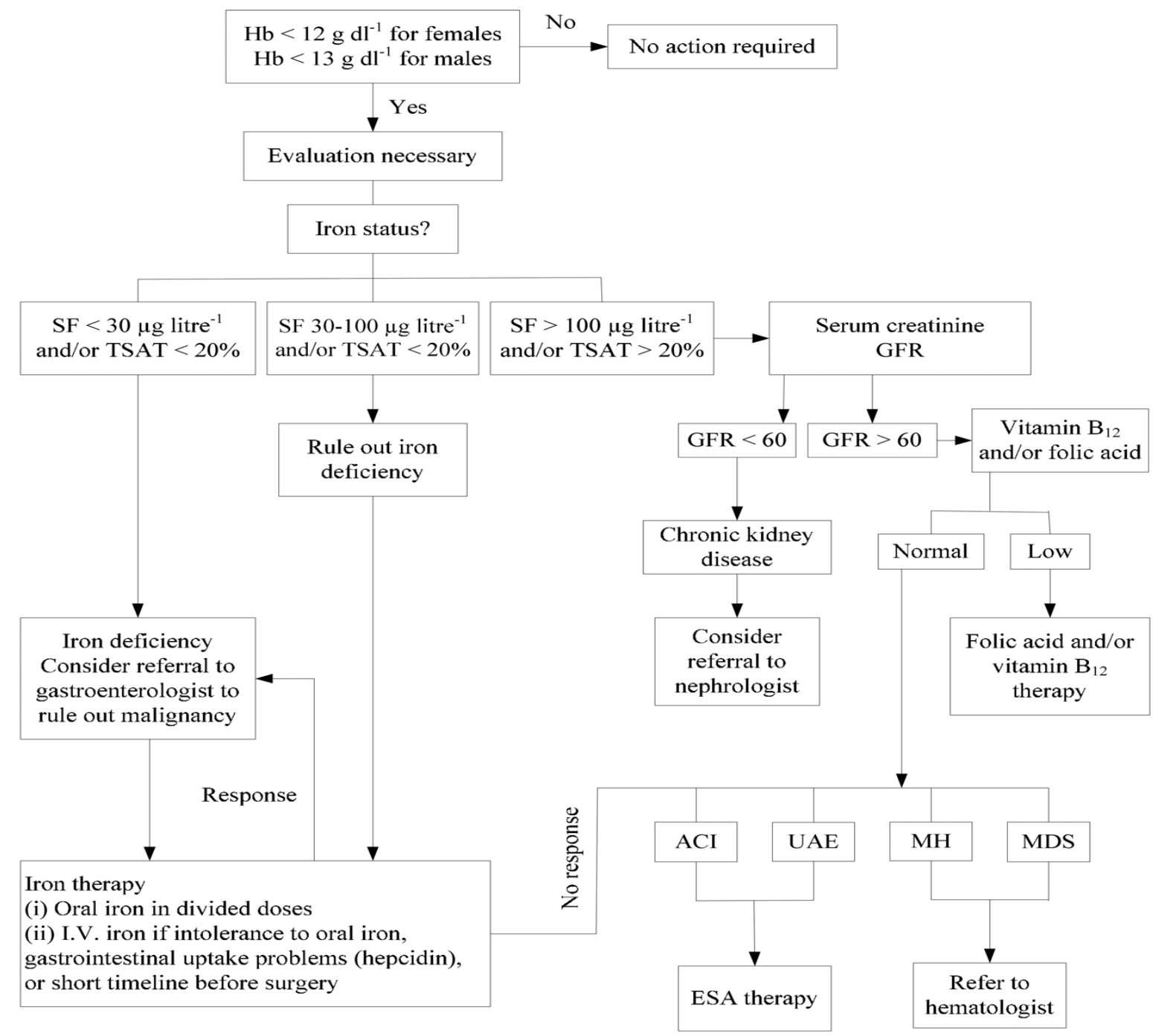

\section{Hb Hemoglobin \\ SF Serum Ferritin \\ GFR Glomerular Filtration Rate \\ ACI Anemia of Inflammation}

UAE Undifferentiated Anemia of the Elderly

MDS Myelodysplastic Syndrome

ESA Erythropoiesis Stimulating Agent

MH Malignant Hematology (e.g. chronic lymphocytic leukemia) 


\section{Appendix A, 1}

On the background above, let us scrutinize the results of the audit; our analysis revealed a very high prevalence of anaemia in the patients admitted to the OPM department of NNUH.

$166(67.2 \%)$ of the 246 patients in the audit were anaemic, this is a very significant proportion but it is in agreement available scientific literature and studies, where it is has been observed that the prevalence of anaemia increases with age as reported above [1-4].

Among anaemic patients only 94 (56.6\%) received further tests to determine the cause of their anaemia (Table 1). This proportion is short of the expected standard of $98 \%$ given the high prevalence of anaemia in the elderly. This indicates that many elderly patients with anaemia are not being properly worked up in the OPM department an NNUH.

Turning to analysis of anaemic patients meeting each criterion (Table 2), $32(34.0 \%)$ patients had their iron level checked (criterion 1), 45 (47.9\%) anaemic patients had their vitamin B12 \& folate checked (criterion 2), and on $17(18.1 \%)$ received ferritin level checks (criterion 3).

These results indicate that nearly half (44\%) anaemic elderly patients admitted at the NNUH OPM department are not receiving any form of investigation for their anaemia once it is detected on the FBC. Vitamin B $12 \&$ folate was the most ordered test, one reason contributing to this I suggest is the fact that as many elderly patients admitted have delirium in addition to other medical problems, B12 \& folate checks are done as part of the delirium screening process and not solely to investigate anaemia.

In summary, from the audit results it is clear that standards are not being met for patients admitted to the OPM department at NNUH, more anaemic patients should receive appropriate subsequent workup for anaemia once identified to have anaemia on admission.

As mentioned above, anaemia even if mild has many serious adverse outcomes, underlying aetiology for the anaemia such as chronic disease, iron deficiency, or myelodysplastic syndromes that can be identified through further investigation.
Changes recommended based on the audit results will include doctors having access to a single anaemia test panel that include haematinics and elemental iron tests as a unit battery of tests.

\section{Plans for change}

There is a need for a single anaemia test panel with all haematinics including elemental Iron to be developed by the laboratory/pathology department so that a doctor can order all appropriate tests in a single click when anaemia is detected in the FBC during admission workup.

For example at Nobles Hospital on the Isle of Man the Iron studies panel includes; Iron, Total Iron Binding, Ferritin, Transferrin Saturation. Could NNUH go further and consider adding vitamin B12 and folate to the Nobles Hospital Iron studies panel? I believe this would represent a robust approach to investigating anaemia in the elderly by a single click of a button when a doctor is ordering investigations.

Also guidelines should be strengthened to make it mandatory for any doctor seeing an elderly anaemic patient to investigate the cause of anaemia or document why they have chosen not to investigate.

\section{References}

1. Guralnik JM, Eisenstaedt RS, Ferrucci L, Klein HG, Woodman RC (2004). Prevalence of anemia in persons 65 years and older in the United States: evidence for a high rate of unexplained anemia. Blood; 104:2263-2268.

2. Zakai NA, French B, Arnold AM, et al (2013). Hemoglobin decline, function, and mortality in the elderly: the Cardiovascular Health Study. Am J Hematol; 88:5-9.

3. Lawrence Tim Goodnough and Stanley L. Schrier Am J Hematol (2014); Evaluation And Management Of Anemia In The Elderly 89(1): 88-96.

4. Bach V, Schruckmayer G, Sam I, Kemmler G, Stauder R. (2014), Prevalence and possible causes of anemia in the elderly: a crosssectional analysis of a large european university hospital cohort, Clin Interv Aging; 9:1187-1196.

5. https://cks.nice.org.uk/anaemia-iron-deficiency\#!diagnosisSub:2

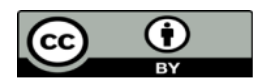

This work is licensed under Creative Commons Attribution 4.0 License
Ready to submit your research? Choose Auctores and benefit from:

$>$ fast, convenient online submission

$>$ rigorous peer review by experienced research in your field

$>$ rapid publication on acceptance

$>$ authors retain copyrights

$>$ unique DOI for all articles

$>$ immediate, unrestricted online access

At Auctores, research is always in progress.

Learn more auctoresonline.org/journals/new-medical-innovations-andresearch 